\title{
Introduction of the chemical potential in the overlap formalism
}

\author{
R. Narayanan \\ Department of Physics, Florida International University, Miami, FL 33199. \\ E-mail: rajamani.narayanan@fiu.edu
}

\author{
Sayantan Sharma \\ Department of Theoretical Physics, Tata Institute of Fundamental Research, Homi \\ Bhabha Road, Mumbai 400005, India \\ E-mail: ssharma@theory.tifr.res.in
}

\begin{abstract}
We investigate the possibility of coupling a chemical potential only to the physical chiral fermions on the lattice starting from the many body state description of overlap fermions. After developing the formalism for a chiral gauge theory, we focus our attention on the case of free fermions coupled to a vector like chemical potential and discuss the issue of zero temperature divergences.
\end{abstract}

KEYwords: Chiral fermions, Chemical potential. 


\section{Contents}

1. Introduction 1

2. The overlap formalism 4

$\begin{array}{lll}\text { 3. Introduction of the chemical potential } & 6\end{array}$

$\begin{array}{lll}\text { 4. Introduction of the fermion mass } & 7\end{array}$

5. Free overlap fermions $\quad 8$

$\begin{array}{ll}\text { 6. Conclusions } & 10\end{array}$

A. Details of the Hermitian Wilson-Dirac operator 14

B. Details of the derivation of generating functional for massive overlap $\begin{array}{ll}\text { fermions with a chemical potential } & 14\end{array}$

\section{Introduction}

Nonperturbative studies of QCD at finite baryon density and finite temperature is an active field of investigation on the lattice with implications for current experimental studies of the transition between the hadronic phase and quark-gluon plasma phase $[1,2,3,4]$. Due to the well known "sign-problem" associated with lattice simulations at finite chemical potential, a large fraction of the numerical simulations compute coefficients in the Taylor expansion in chemical potential $[5,6]$. Typically one computes susceptibilities (second order coefficients) but higher moments have also been computed [3, 7]. The Taylor expansion is expected to diverge close to the critical point where fluctuations dominate the physical behavior [8].

We will focus on the quark number susceptibility for definiteness in this paper but the formalism in this paper carries over to all coefficients in a Taylor expansion. Let $Z\left(\mu, N_{T}\right)$ be the grand canonical partition function on a $N^{3} \times N_{T}$ lattice at a given chemical potential $\mu$. The dependence of the partition function on other quantities like gauge coupling, quark masses etc. have been suppressed. We also assume that we will take the thermodynamic limit, $N \rightarrow \infty$, and $N_{T}^{-1}$ will play the role of temperature on the lattice. The quark number susceptibility is defined as

$$
\chi\left(N_{T}\right)=\left.\lim _{N \rightarrow \infty} \frac{1}{N^{3} N_{T}} \frac{\partial^{2} \ln Z\left(\mu, N_{T}\right)}{\partial \mu^{2}}\right|_{\mu=0} .
$$


This quantity has been studied both on the lattice [1,9] and in other non-perturbative approaches [10].

A näive introduction of the number density operator on the lattice leads to divergences at zero temperature in the following sense. A proper definition should yield a result that goes as $\frac{1}{N_{T}^{2}}$ and the presence of a non-zero $\chi(\infty)$ implies a divergence in the continuum limit. Such a divergence can be removed by replacing $U_{4}(x)$ and $U_{4}^{\dagger}(x)$ on the lattice by $e^{\mu} U_{4}(x)$ and $e^{-\mu} U_{4}^{\dagger}(x)$ where $U_{4}(x)$ is the link variable on the lattice connecting $x$ and $x+\hat{4}[11]$ and will be referred as the Hasenfratz-Karsch (H-K) prescription. Let $T_{4}$ denote operator

$$
T_{4} \psi(x)=U_{4}(x) \psi(x+\hat{4}) .
$$

For näive fermions, the source term added to the action would be

$$
j_{4}= \begin{cases}\bar{\psi} \gamma_{4} \psi & \text { for näive insertion; } \\ \bar{\psi} \gamma_{4} \frac{\left(e^{\mu}-1\right) T_{4}-\left(e^{-\mu}-1\right) T_{4}^{\dagger}}{2} \psi & \text { for H-K insertion }\end{cases}
$$

The näive insertion would result in a non-zero $\chi(\infty)$ but the in H-K insertion it would lead off as $\frac{1}{N_{T}^{2}}$.

The aim of this paper is to address the introduction of the chemical potential into the overlap Dirac operator [12]. It was introduced using the H-K prescription by Bloch and Wettig [13]. The Wilson-Dirac operator, $H_{w}(\mu)$, that appears as the kernel in the definition of the overlap Dirac operator is not Hermitian in the presence of a chemical potential. The massless overlap Dirac operator in the presence of the chemical potential as per the H-K prescription is

$$
D_{\mathrm{BW}}(\mu)=\frac{1+\gamma_{5} \epsilon\left(H_{w}(\mu)\right)}{2} .
$$

Assuming $H_{w}(\mu)$ is diagonalizable in the form

$$
H_{w}(\mu)=R \Lambda R^{-1}
$$

with $\Lambda$ being a diagonal matrix with complex entries, one sets

$$
\epsilon\left(H_{w}(\mu)\right)=\operatorname{R\epsilon }(\operatorname{Re} \Lambda) R^{-1}
$$

Such a choice can be justified using the domain wall formalism [14] since $\epsilon\left(H_{w}\right)$ is the limit of $\tanh \left(L_{s} H_{w}\right)$ with $L_{s}$ being the extent in the extra continuous dimension [15]. In spite of the fact that $V(\mu)=\gamma_{5} \epsilon\left(H_{w}(\mu)\right)$ is not a unitary operator for $\mu \neq 0$, the massless propagator defined in the usual manner $[15,16]$ as

$$
G(\mu)=\frac{1-V(\mu)}{1+V(\mu)}
$$

is still chiral: Let us write

$$
R=\left(\begin{array}{cc}
\alpha & \gamma \\
\beta & \delta
\end{array}\right) ; \quad \Lambda=\left(\begin{array}{cc}
\Lambda^{+} & 0 \\
0 & -\Lambda^{-}
\end{array}\right) ; \quad \operatorname{Re} \Lambda^{ \pm}>0
$$


Then one can show that

$$
G(\mu)=\left(\begin{array}{cc}
0 & \gamma \delta^{-1} \\
\beta \alpha^{-1} & 0
\end{array}\right)
$$

showing the chiral nature of the massless propagator assuming that $\alpha$ and $\delta$ are invertible complex matrices. Both the energy density [17] and the quark number susceptibility [18] for the case of free overlap fermions were analyzed within the Bloch-Wettig scheme and shown to have the correct continuum behavior. One possible objection to the Bloch-Wettig scheme would be that the chemical potential is coupled to all the fermions from the viewpoint of domain wall fermions and not just to the chiral fermions. This objection could also be raised for Wilson fermions where the chemical potential is coupled to the massless mode as well as the massive doublers but we should keep in mind that we have infinite number of fermions in the case of domain wall fermions. Since we have an explicit operator for the physical fermions, it should be possible to couple the chemical potential just to the physical fermions in the overlap formalism. Another possible objection already raised in [13] is that the number of topological modes can depend on the chemical potential since the topological charge is

$$
Q=\int d^{4} x q(x)=\frac{1}{2} \int d^{4} x \operatorname{tr} \epsilon(x),
$$

where tr is the trace over the spin components at a fixed point $x$. Since $\epsilon\left(H_{w}(\mu)\right)$ depends on $\mu, Q$ can change with $\mu$ in the Bloch-Wettig scheme. In fact $q(x)$ was shown to depend on $\mu$ in [19].

We will start from the first principles of the overlap formalism [20] and add a chemical potential by coupling it to the number density operator. We will consider the general case where the chemical potential for the fermions with positive and negative chirality can be different. We will make a class of choices for the number density operator all of which reduce to the standard continuum limit. We will focus on the case of free overlap Dirac fermions and show that divergences in the quark number susceptibility is a generic feature. Since every member in our class of choices will contain the correct $\frac{1}{3 N_{T}^{2}}$ in the free quark number susceptibility, we will be able to write down a large class of number density operators that will not contain a divergent piece.

As a first step, we will review the generating functional ${ }^{1}$ for a massless vector like gauge theory. None of this is new and can be found in one form or other in $[15,20]$. In particular, we will explicitly write down the chiral transformations in the overlap formalism and derive the massless overlap Dirac operator [12]. This will help us introduce the chemical potential and derive the associated generating functional for the massless overlap Dirac operator with a chemical potential. The resulting class of operators will be different from $D_{\mathrm{BW}}(\mu)$ in (1.4) but the generating functional will be invariant under chiral transformations. All these operators will have two features that separate them from the operator in the BlochWettig scheme: Chemical potential will only be coupled to the physical chiral fermions and topological charge will not depend on the chemical potential. Finally, we will discuss the

\footnotetext{
${ }^{1}$ We will only consider the generating functional for a fixed gauge field background through out this paper.
} 
addition of a mass term to the generating functional that destroys the chiral symmetry. We will end the paper with a discussion of the quark number susceptibility for free overlap Dirac fermions arising from our generating functional.

\section{The overlap formalism}

This section is essentially a repeat of what can be found in [20]. The main difference is that we only use one Hermitian Wilson-Dirac operator in the construction of overlap fermions. This amounts to setting the Wilson mass parameter to infinity on the inactive side. This changes the details as it pertains to the generating functional and since we will derive the generating functional for massive overlap fermions in the presence of the chemical potential, we will need explicit results from this section.

At the heart of the overlap formalism is the massive Hermitian Dirac operator which is usually realized on the lattice using the Wilson-Dirac operator, $H_{w}$. The notational details are given in Appendix A. The generating functional for a vector like theory with massless fermions is given by [20]

$$
Z(\bar{\xi}, \xi)={ }_{R}\left\langle-\left|e^{\bar{\xi}_{R} d_{R}+\xi_{R} u_{R}^{\dagger}}\right|+\right\rangle_{R} \quad{ }_{L}\left\langle+\left|e^{\xi_{L} d_{L}^{\dagger}+\bar{\xi}_{L} u_{L}}\right|-\right\rangle_{L} .
$$

Dirac valued operators, $a_{R, L}, a_{R, L}^{\dagger}$, obey canonical anti-commutation relations separately for the $R$ and $L$ sets. We write,

$$
a_{R, L}=\left(\begin{array}{c}
u_{R, L} \\
d_{R, L}
\end{array}\right)
$$

in terms of their respective Weyl components. The sources,

$$
\bar{\xi}=\left(\begin{array}{ll}
\bar{\xi}_{R} & \bar{\xi}_{L}
\end{array}\right) ; \quad \xi=\left(\begin{array}{l}
\xi_{R} \\
\xi_{L}
\end{array}\right)
$$

are Dirac values Grassmann variables that couple directly to the physically relevant fields and they anti-commute with the fermionic operators. $|+\rangle_{R, L}$ are lowest states of

$$
\mathcal{H}_{R, L}=-a_{R, L}^{\dagger} H_{w} a_{R, L}
$$

and $|-\rangle_{R, L}$ are lowest states of two many body operators, ${ }^{2}$

$$
\Gamma_{R, L}=-a_{R, L}^{\dagger} \gamma_{5} a_{R, L}
$$

We now make several remarks on the generating functional.

1. The phase choice for $|+\rangle_{R, L}$ are tied together since they are the ground states of identical many body operators. The same is true for $|-\rangle_{R, L}$. Therefore, the generating functional is unambiguous and does not not depend upon the phase choice present in the unitary matrix, $U$, that diagonalizes $H_{w}$.

\footnotetext{
${ }^{2}$ The pair $\mathcal{H}_{R}$ and $\Gamma_{R}$ replaces the $\mathbf{H}_{ \pm}$in [20]. That one can take the Wilson mass parameter to infinity on one side follows from the discussion of the topological charge in section 8 of [20].
} 
2. It does not depend on the ordering of the operators since the two terms in the exponent commute with each other in both factors.

3. It is clear that $d_{R}$ and $u_{R}^{\dagger}$ are the propagating degrees of freedom in the first factor since ${ }_{R}\langle-| u_{R}$ and ${ }_{R}\langle-| d_{R}^{\dagger}$ are both zero. The converse holds for the second factor.

4. The generating functional is invariant under global chiral transformations:

$$
\xi_{R} \rightarrow e^{i \varphi_{R}} \xi_{R} ; \quad \bar{\xi}_{R} \rightarrow \bar{\xi}_{R} e^{-i \varphi_{R}} ; \quad \xi_{L} \rightarrow e^{i \varphi_{L}} \xi_{L} ; \quad \bar{\xi}_{L} \rightarrow \bar{\xi}_{L} e^{-i \varphi_{L}}
$$

5. The explicit expression for the generating functional can be obtained by small modifications to the details presented in [20] and the result is

$$
Z(\bar{\xi}, \xi)=\left[e^{\bar{\xi}_{R} \beta \alpha^{-1} \xi_{R}} \operatorname{det} \alpha\right]\left[e^{\bar{\xi}_{L}\left[\beta \alpha^{-1}\right]^{\dagger} \xi_{L}} \operatorname{det} \alpha^{\dagger}\right]
$$

In particular, the propagators for right-handed and left-handed fermions are

$$
G_{R}^{i j}=\frac{{ }_{R}\left\langle-\left|u_{R j}^{\dagger} d_{R i}\right|+\right\rangle_{R}}{{ }_{R}\langle-\mid+\rangle_{R}}=\left[\beta \alpha^{-1}\right]_{i j} ; \quad G_{L}^{i j}=\frac{{ }_{L}\left\langle+\left|d_{L j}^{\dagger} u_{L i}\right|-\right\rangle_{L}}{{ }_{L}\langle+\mid-\rangle_{L}}=\left[\beta \alpha^{-1}\right]_{i j}^{\dagger},
$$

and they obey the relation

$$
G_{R}^{\dagger}=G_{L}
$$

In practice, one can avoid exact diagonalization of $H_{w}$ which is needed for the computation of $U$ and (2.7) since one has an overlap-Dirac operator for vector like theories [12]. Consider the unitary operator,

$$
V=\gamma_{5} \epsilon\left(H_{w}\right)
$$

It follows from (A.6) and (A.7) that

$$
\frac{1+V}{2} U=\left(\begin{array}{cc}
\alpha & 0 \\
0 & \delta
\end{array}\right) ; \quad \frac{1-V}{2} U=\left(\begin{array}{cc}
0 & \gamma \\
\beta & 0
\end{array}\right),
$$

and therefore

$$
G_{o}=\frac{1-V}{1+V}=\left(\begin{array}{cc}
0 & -\left(\beta \alpha^{-1}\right)^{\dagger} \\
\beta \alpha^{-1} & 0
\end{array}\right),
$$

is the massless overlap Dirac propagator. Since [20]

$$
\operatorname{det} U=\frac{\operatorname{det} \alpha}{\operatorname{det} \delta^{\dagger}} ;
$$

we have the identity,

$$
\operatorname{det} \alpha \operatorname{det} \alpha^{\dagger}=\operatorname{det} \delta \operatorname{det} \delta^{\dagger} \text {, }
$$

and therefore,

$$
\operatorname{det} \frac{1+V}{2}=\operatorname{det} \alpha \operatorname{det} \alpha^{\dagger} .
$$

The massless overlap Dirac operator is given by

$$
D_{o}=\frac{1+V}{2} .
$$


Our generating functional in $(2.7)$ can be written as ${ }^{3}$

$$
Z(\bar{\xi}, \xi)=\operatorname{det} D_{o} e^{-i \bar{\xi} \gamma_{4} G_{0} \xi}
$$

We can use (2.12) and (2.15) along with an efficient implementation of $V$ to compute the generating functional. Note that the operator in (2.16) is not identical to the operator used to compute the propagator in (2.12). This is an essential ingredient of the overlap formalism.

\section{Introduction of the chemical potential}

Consider the generating functional

$$
Z\left(\bar{\xi}, \xi ; \hat{\mu}_{R}, \hat{\mu}_{L}\right)={ }_{R}\left\langle-\left|e^{\bar{\xi}_{R} d_{R}+\xi_{R} u_{R}^{\dagger}+u_{R}^{\dagger} \hat{\mu}_{R} d_{R}}\right|+\right\rangle_{R L}\left\langle+\left|e^{\xi_{L} d_{L}^{\dagger}+\bar{\xi}_{L} u_{L}-d_{L}^{\dagger} \hat{\mu}_{L} u_{L}}\right|-\right\rangle_{L},
$$

where $\hat{\mu}_{R}\left(\mu_{R}\right)$ and $\hat{\mu}_{L}\left(\mu_{L}\right)$ are operators that parametrically depend on the chemical potentials, $\mu_{R}$ and $\mu_{L}$.

In order to obtain a formula for the generating functional, we start by noting that

$$
\int d \zeta_{R} d \bar{\zeta}_{R} e^{-\bar{\zeta}_{R} \hat{\mu}_{R} d_{R}-\zeta_{R} u_{R}^{\dagger}+\bar{\zeta}_{R} \zeta_{R}}=e^{u_{R}^{\dagger} \hat{\mu}_{R} d_{R}}
$$

and

$$
\int d \bar{\zeta}_{L} d \zeta_{L} e^{-\bar{\zeta}_{L} \hat{\mu}_{L} u_{L}-\zeta_{L} d_{L}^{\dagger}-\bar{\zeta}_{L} \zeta_{L}}=e^{-d_{L}^{\dagger} \hat{\mu}_{L} u_{L}}
$$

where $\zeta_{R}, \zeta_{L}, \bar{\zeta}_{R}, \bar{\zeta}_{L}$ are Grassmann variables that anticommute with all fermionic operators and Grassmann variables. Therefore (2.1) and (2.7) gets modified to

$$
\begin{aligned}
Z\left(\bar{\xi}, \xi ; \mu_{R}, \mu_{L}\right)= & \operatorname{det} \alpha \operatorname{det} \alpha^{\dagger} \\
& \int d \zeta_{R} d \bar{\zeta}_{R} e^{\bar{\zeta}_{R} \zeta_{R}+\left(\bar{\xi}_{R}-\bar{\zeta}_{R} \hat{\mu}_{R}\right) \beta \alpha^{-1}\left(\xi_{R}-\zeta_{R}\right)} \\
& \int d \bar{\zeta}_{L} d \zeta_{L} e^{-\bar{\zeta}_{L} \zeta_{L}+\left(\bar{\xi}_{L}-\bar{\zeta}_{L} \hat{\mu}_{L}\right)\left[\beta \alpha^{-1}\right]^{\dagger}\left(\xi_{L}-\zeta_{L}\right)} \\
= & \operatorname{det} \alpha \operatorname{det}\left(1+\hat{\mu}_{R} \beta \alpha^{-1}\right) \operatorname{det} \alpha^{\dagger} \operatorname{det}\left(1-\hat{\mu}_{L}\left[\beta \alpha^{-1}\right]^{\dagger}\right) \\
& e^{\bar{\xi}_{R} \frac{1}{\alpha \beta^{-1}+\hat{\mu}_{R}} \xi_{R}} e^{\bar{\xi}_{L} \frac{1}{\left[\alpha \beta^{-1}\right]^{\dagger}-\hat{\mu}_{L}} \xi_{L}} .
\end{aligned}
$$

Note that the generating functional is invariant under the chiral transformation given in (2.6) since the introduction of the chemical potential does not mix the two chiral sectors. To be consistent with the continuum definition of the chemical potentials, we require

$$
\hat{\mu}_{R}(0)=0 ; \quad \hat{\mu}_{L}(\mu)=-\hat{\mu}_{R}^{\dagger}(-\mu) .
$$

Note that the fermion determinant is not real and positive for a real quark chemical potential, $\mu_{R}=\mu_{L}=\mu$, but it is real and positive for an isospin chemical potential, $\mu_{R}=$ $-\mu_{L}=\mu$. These are standard properties in the continuum that are correctly reproduced by the overlap formalism.

\footnotetext{
${ }^{3}$ The presence of $-i \gamma_{4}$ is due to our choice of basis for the chiral sources.
} 
There are several options for $\hat{\mu}_{R}(\mu)$. We will address this issue when we analyze free fermions.

We can write (3.4) in a compact form by introducing the massless overlap Dirac operator and propagator in the presence of a chemical potential. If we define

$$
\begin{aligned}
N & =\left(\begin{array}{cc}
0 & \hat{\mu}_{R} \\
\hat{\mu}_{L} & 0
\end{array}\right) ; \\
D_{o}\left(\hat{\mu}_{R}, \hat{\mu}_{L}\right) & =\frac{1+V}{2}+N \frac{1-V}{2} \\
G_{o}\left(\hat{\mu}_{R}, \hat{\mu}_{L}\right) & =\left[\frac{1+V}{1-V}+N\right]^{-1} .
\end{aligned}
$$

then a straight forward computation shows that

$$
Z\left(\bar{\xi}, \xi ; \hat{\mu}_{R}, \hat{\mu}_{L}\right)=\operatorname{det} D_{o}\left(\hat{\mu}_{R}, \hat{\mu}_{L}\right) e^{-i \bar{\xi} \gamma_{4} G_{o}\left(\hat{\mu}_{R}, \hat{\mu}_{L}\right) \xi} .
$$

\section{Introduction of the fermion mass}

A mass term can be added in analogy with the chemical potential term [20]. The generating functional in the presence of a chemical potential and mass term is defined to be

$$
\begin{aligned}
& Z\left(\bar{\xi}, \xi ; \hat{\mu}_{R}, \hat{\mu}_{L} ; m\right)=\left[{ }_{R}\langle-| \otimes_{L}\langle+|\right] \\
& e^{\bar{\xi}_{R} d_{R}+\xi_{R} u_{R}^{\dagger}+u_{R}^{\dagger} \hat{\mu}_{R} d_{R}+\xi_{L} d_{L}^{\dagger}+\bar{\xi}_{L} u_{L}-d_{L}^{\dagger} \hat{\mu}_{L} u_{L}+m d_{L}^{\dagger} d_{R}-m u_{R}^{\dagger} u_{L}} \\
& {\left[|-\rangle_{L} \otimes|+\rangle_{R}\right]}
\end{aligned}
$$

One can show using methods similar to one in (3) (see Appendix B for details) that

$$
\begin{aligned}
& Z\left(\bar{\xi}, \xi ; \hat{\mu}_{R}, \hat{\mu}_{L} ; m\right) \\
= & \operatorname{det} \alpha \operatorname{det}\left(1+\hat{\mu}_{R} \beta \alpha^{-1}\right) \operatorname{det} \alpha^{\dagger} \operatorname{det}\left(1-\hat{\mu}_{L}\left(\beta \alpha^{-1}\right)^{\dagger}\right) \\
& \operatorname{det}\left[1+m^{2} \frac{1}{\alpha \beta^{-1}+\hat{\mu}_{R}}\left[\frac{1}{\left(\alpha \beta^{-1}\right)^{\dagger}-\hat{\mu}_{L}}\right]\right] \\
& \bar{\xi}_{R}\left(\left(\alpha \beta^{-1}\right)^{\dagger}-\hat{\mu}_{L}\right) \frac{1}{m^{2}+\left(\alpha \beta^{-1}+\hat{\mu}_{R}\right)\left(\left(\alpha \beta^{-1}\right)^{\dagger}-\hat{\mu}_{L}\right)} \xi_{R}+\bar{\xi}_{L}\left(\alpha \beta^{-1}+\hat{\mu}_{R}\right) \frac{m}{m^{2}+\left(\left(\alpha \beta^{-1}\right)^{\dagger}-\hat{\mu}_{L}\right)\left(\alpha \beta^{-1}+\hat{\mu}_{R}\right)} \xi_{L} \\
& e_{\bar{\xi}_{R} \frac{m}{m^{2}+\left(\left(\alpha \beta^{-1}\right)^{\dagger}-\hat{\mu}_{L}\right)\left(\alpha \beta^{-1}+\hat{\mu}_{R}\right)} \xi_{L}-\bar{\xi}_{L} \frac{m^{2}+\left(\alpha \beta^{-1}+\hat{\mu}_{R}\right)\left(\left(\alpha \beta^{-1}\right)^{\dagger}-\hat{\mu}_{L}\right)}{m_{R}}}^{\xi^{2}} .
\end{aligned}
$$

We can write (4.2) in a compact form by extending the massless overlap Dirac operator in the presence of a chemical potential define in (3.6) to include a mass as follows: If we define

$$
\begin{aligned}
N(m) & =\left(\begin{array}{cc}
m & \hat{\mu}_{R} \\
\hat{\mu}_{L} & m
\end{array}\right) \\
D_{o}\left(\hat{\mu}_{R}, \hat{\mu}_{L} ; m\right) & =\frac{1+V}{2}+N(m) \frac{1-V}{2} \\
G_{o}\left(\hat{\mu}_{R}, \hat{\mu}_{L} ; m\right) & =\left[\frac{1+V}{1-V}+N(m)\right]^{-1} .
\end{aligned}
$$

then a straight forward computation show that

$$
Z\left(\bar{\xi}, \xi ; \hat{\mu}_{R}, \hat{\mu}_{L} ; m\right)=\operatorname{det} D_{o}\left(\hat{\mu}_{R}, \hat{\mu}_{L} ; m\right) e^{-i \bar{\xi} \gamma_{4} G_{o}\left(\hat{\mu}_{R}, \hat{\mu}_{L} ; m\right) \xi}
$$

Note that the expressions reduce to the usual ones [16] for the massive overlap Dirac operator without a chemical potential. 


\section{Free overlap fermions}

In order to work out the energy density and quark number susceptibility for free massless quarks, it is best to work in momentum space. Let us assume that we have converted to creation and annihilation operators in momentum space by the appropriate unitary transformation. We will work on a $\infty^{3} \times N_{T}$ lattice. The allowed spatial momenta are $p_{k} \in[-\pi, \pi]$. The Matsubara frequencies in the $N_{T}$ direction are $\omega_{n}= \pm \frac{2 \pi n+\pi}{N_{T}} ; n=$ $0, \cdots, \frac{N_{T}}{2}-1$ and we will assume that $N_{T}$ is even. The hermitian Wilson-Dirac operator for a fixed momentum takes the form

$$
H_{w}=\left(\begin{array}{cc}
w & c \\
c^{\dagger} & -w
\end{array}\right)
$$

where

$$
\begin{gathered}
w=2 \sum_{k} \sin ^{2} \frac{p_{k}}{2}+2 \sin ^{2} \frac{\omega_{n}}{2}-m_{w} ; \quad c=i \sum_{k} \sigma_{k} \sin p_{k}-\sin \omega_{n} \\
c c^{\dagger}=c^{\dagger} c=\sum_{k} \sin ^{2} p_{k}+\sin ^{2} \omega_{n} .
\end{gathered}
$$

The positive eigenvalues come in one doubly degenerate pair, $\lambda=\sqrt{w^{2}+c c^{\dagger}}$, per momentum block and the corresponding pair of orthonormal eigenvectors are

$$
\frac{1}{\sqrt{2 \lambda(\lambda-w)}}\left(\begin{array}{c}
c \\
\lambda-w
\end{array}\right)
$$

Note that $\lambda$ and $w$ are even functions of $\omega_{n}$ at a fixed $p_{k}$. Assuming a vector like chemical potential, we write

$$
N=\left(\begin{array}{cc}
0 & n\left(\mu, \omega_{n}\right) \\
-n^{*}\left(-\mu, \omega_{n}\right) & 0
\end{array}\right)
$$

per momentum block where $n\left(0, \omega_{n}\right)=0$. We write,

$$
n\left(\mu, \omega_{n}\right)=n_{r}\left(\mu, \omega_{n}\right)+i n_{i}\left(\mu, \omega_{n}\right)
$$

where $n_{r, i}\left(\mu, \omega_{n}\right)$ are both real functions. The free energy density is given by

$$
\ln Z\left(\mu, N_{T}\right)=\prod_{i=1}^{3} \int_{-\pi}^{\pi} \frac{d p_{i}}{2 \pi} \sum_{n}\left\{-2 \ln (2 \lambda)+\ln \left(g^{2}+h\right)\right\}
$$

where

$$
g=\lambda+w+n\left(\mu, \omega_{n}\right) n^{*}\left(-\mu, \omega_{n}\right)(\lambda-w)-\left[n\left(\mu, \omega_{n}\right)+n^{*}\left(-\mu, \omega_{n}\right)\right] \sin \omega_{n},
$$

and

$$
h=\left[n\left(\mu, \omega_{n}\right)-n^{*}\left(-\mu, \omega_{n}\right)\right]^{2} \sum_{k=1}^{3} \sin ^{2} p_{k} .
$$


The quark number susceptibility is

$\chi\left(N_{T}, m_{w}\right)=\frac{1}{N_{T}} \prod_{i=1}^{3} \int_{-\pi}^{\pi} \frac{d p_{i}}{2 \pi} \sum_{n} \frac{4\left(q^{2}-r^{2}\right)\left(\sin ^{2} \omega_{n}-\sum_{k=1}^{3} \sin ^{2} p_{k}\right)-4 s\left(\omega_{n}\right)(\lambda+w) \sin \omega_{n}}{(\lambda+w)^{2}}$,

where

$$
q\left(\omega_{n}\right)=\left.\frac{\partial n_{i}\left(\mu, \omega_{n}\right)}{\partial \mu}\right|_{\mu=0} ; \quad r\left(\omega_{n}\right)=\left.\frac{\partial n_{r}\left(\mu, \omega_{n}\right)}{\partial \mu}\right|_{\mu=0} ; \quad s\left(\omega_{n}\right)=\left.\frac{\partial^{2} n_{r}\left(\mu, \omega_{n}\right)}{\partial^{2} \mu}\right|_{\mu=0} .
$$

The sum over $n$ can be non-zero only if $4\left(q^{2}-r^{2}\right)$ is an even function of $\omega_{n}$ and $s$ is an odd function of $\omega_{n}$.

Two choices one typically makes are

$$
\hat{\mu}_{R}(\mu)=\frac{i}{2 m_{w}} \begin{cases}\mu & \text { for näive insertion; } \\ \frac{\left(e^{\mu}-1\right) T_{4}-\left(e^{-\mu}-1\right) T_{4}^{\dagger}}{2} & \text { for H-K insertion; }\end{cases}
$$

resulting in

$$
n\left(\mu, \omega_{n}\right)=\frac{1}{2 m_{w}} \begin{cases}i \mu & \text { for näive insertion; } \\ \sin \omega_{n}-\sin \left(\omega_{n}-i \mu\right) & \text { for H-K insertion. }\end{cases}
$$

The $\frac{1}{m_{w}}$ factor comes from the tree-level wavefunction renormalization [16]. For the näive insertion,

$$
q=\frac{1}{2 m_{w}} ; \quad r=0 ; \quad s=0
$$

For the H-K insertion,

$$
q=\frac{\cos \omega_{n}}{2 m_{w}} ; \quad r=0 ; \quad s=-\frac{\sin \omega_{n}}{2 m_{w}}
$$

We can make a modification to the H-K insertion such that $s=0$ but keep $q$ to be the same. This corresponds to

$$
\hat{\mu}_{R}(\mu)=\frac{i}{2 m_{w}} \sinh \frac{\mu}{2}\left(T_{4}+T_{4}^{\dagger}\right) \Rightarrow n\left(\mu, \omega_{n}\right)=\frac{i}{m_{w}} \cos \omega_{n} \sinh \frac{\mu}{2} .
$$

Keeping only the forward derivative will result in

$$
n\left(\mu, \omega_{n}\right)=\frac{i}{m_{w}} e^{i \omega_{n}} \sinh \frac{\mu}{2},
$$

with

$$
q=\frac{\cos \omega_{n}}{2 m_{w}} ; \quad r=-\frac{\sin \omega_{n}}{2 m_{w}} ; \quad s=0 .
$$

This leads us to consider the restricted class of operators,

$$
\hat{\mu}_{R}^{j}(\mu)=\frac{i}{m_{w}} \sinh \frac{\mu}{2} T_{4}^{j} \Rightarrow n^{j}\left(\mu, \omega_{n}\right)=\frac{i}{m_{w}} e^{i j \omega_{n}} \sinh \frac{\mu}{2},
$$


with

$$
q=\frac{\cos j \omega_{n}}{2 m_{w}} ; \quad r=-\frac{\sin j \omega_{n}}{2 m_{w}} ; \quad s=0 ; \quad q^{2}-r^{2}=\frac{\cos 2 j \omega_{n}}{\left(2 m_{w}\right)^{2}} .
$$

The operator $T_{4}^{j}$ is the $\mathrm{j}$-th power of the operator $T_{4}$ defined in (1.2). This motivates us to study

$$
I_{j}\left(N_{T}, m_{w}\right)=\frac{1}{N_{T} m_{w}^{2}} \prod_{i=1}^{3} \int_{-\pi}^{\pi} \frac{d p_{i}}{2 \pi} \sum_{n} \frac{\cos \left(j \omega_{n}\right)\left(\sin ^{2} \omega_{n}-\sum_{k=1}^{3} \sin ^{2} p_{k}\right)}{(\lambda+w)^{2}}, j=\text { even } .
$$

and we conjecture that

$$
I_{j}\left(N_{T}, m_{w}\right)=I_{j}\left(\infty, m_{w}\right)+\frac{1}{3 N_{T}^{2}}+O\left(N_{T}^{-4}\right) .
$$

We are unable to prove (5.21) analytically since we need to work at a finite but large $N_{T}$. The resulting contour integral is complicated due to the presence of the branch cuts associated with square roots signs in definition of $\lambda$. We resort to a numerical check of the conjecture. For this purpose, we convert the three dimensional integral over the momenta to a three dimensional sum with $N$ points in each direction. Let $I_{j}\left(N, N_{T}, m_{w}\right)$ denote this sum. Keeping $N$ fixed, we compute the $I_{j}\left(N, \infty, m_{w}\right)$ by computing the quantity for a very large $N_{T}$. We take this result and extrapolate to $N \rightarrow \infty$ and obtain $I_{j}\left(\infty, m_{w}\right)$. We then consider $I_{j}\left(\zeta N_{T}, N_{T}, m_{w}\right)-I_{j}\left(\infty, m_{w}\right)$ with $\zeta=3,4$ in order to extract the $\frac{1}{N_{T}^{2}}$ coefficient in (5.21). We numerically evaluated

$$
R_{j}\left(\zeta, N_{T}, m_{w}\right)=3 N_{T}^{2}\left(I_{j}\left(\zeta N_{T}, N_{T}, m_{w}\right)-I_{j}\left(\infty, m_{w}\right)\right)-1
$$

for $j=2,4$ at $\zeta=3,4$ and $m_{w}=1,1.5$. It should approach zero with corrections of the order of $\frac{1}{N_{T}^{2}}$. Fig. 1 shows numerical evidence in support of the conjecture: The fits show that $R_{j}\left(4, N_{T}, 1\right)$ approach zero as $\frac{1}{N_{T}^{2}}$ but the corrections get larger with $j$. Fig. 2 shows that $\zeta=4$ is sufficiently large for the numerical computation at hand and Fig. 3 shows that the effect of the Wilson mass, after taking into account the tree level wavefunction renormalization factor, is minimal.

\section{Conclusions}

We have discussed the problem of introducing the chemical potential within the overlap formalism. The formalism presented here couples the chemical potential only to the physical chiral fermions and the overlap definition of the topological charge is unaffected by the value of the chemical potential on the lattice. We have discussed a large class of operators coupled to the chemical potential. Each one of them results in a divergent quark number susceptibility but all of them have the correct continuum limit after the divergent contribution is subtracted. We envision using the formalism developed in this paper in two ways: 


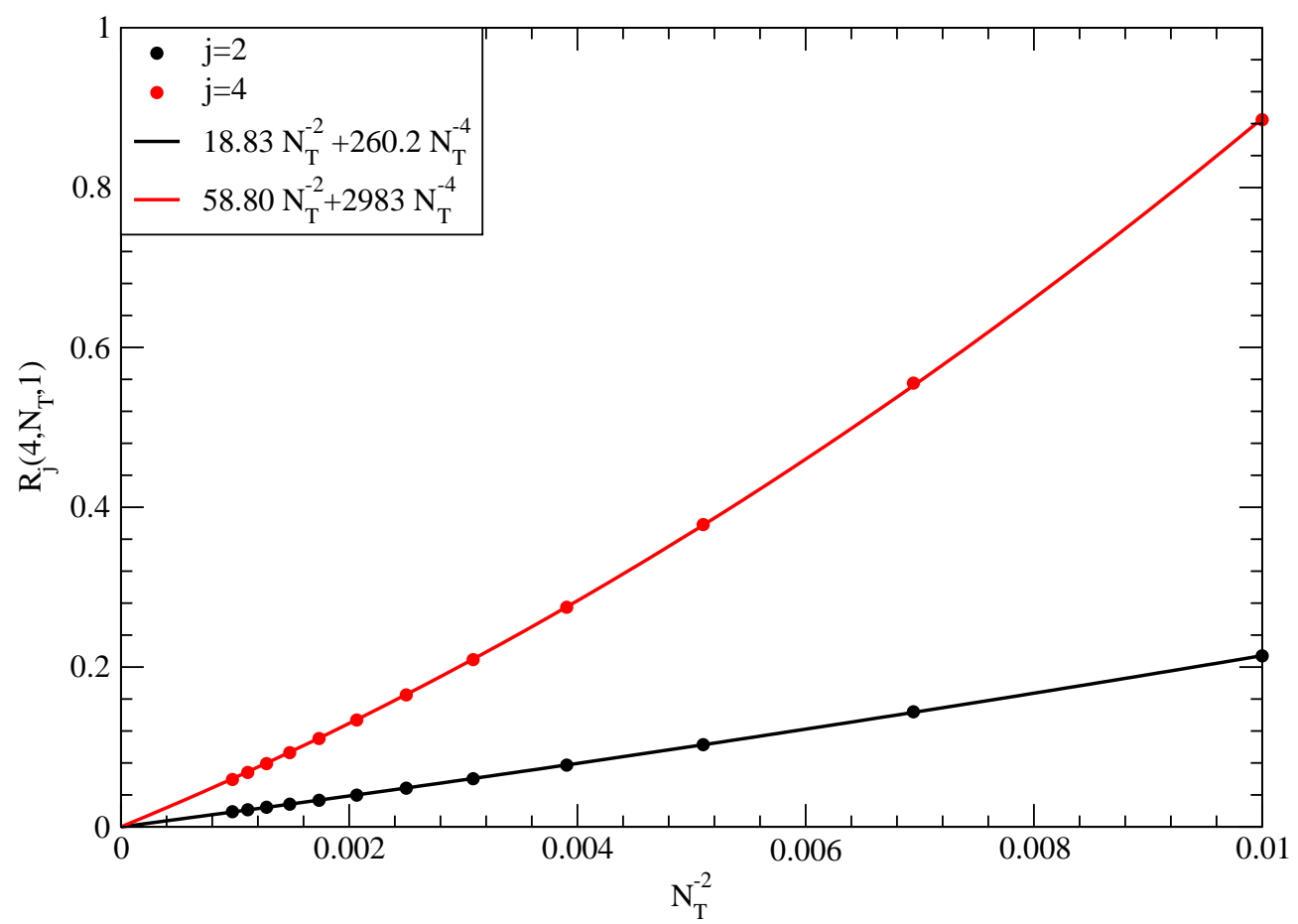

Figure 1: Numerically obtained plot in support of the conjecture.

1. Since the main physics aim in the Taylor expansion method of studying the phase transition from the hadronic phase to the quark-gluon plasma phase is to look for growing fluctuations close to the phase transition, we could consider, say the quark number susceptibility, by taking the difference between its value on a finite temperature lattice and zero temperature lattice for the same value of lattice gauge couplings and lattice quark masses [19]. This will remove the zero temperature divergences and enable a proper study of the fluctuations close to the transition temperature. The chemical potential was coupled to only those physical fermions that are confined to the $4 \mathrm{D}$ domain wall at the origin of the fifth dimension in [19]. However the number density term did not commute with the overlap Hamiltonian. In this paper the chemical potential is coupled to the conserved number density operator explicit in the many body overlap formalism.

2. Consider the linear combination,

$$
\hat{\mu}_{R}(\mu)=\sum_{j=1}^{\infty} c_{j} \hat{\mu}_{R}^{j}(\mu) .
$$

The associated free fermion quark number susceptibility for this choice of the number 


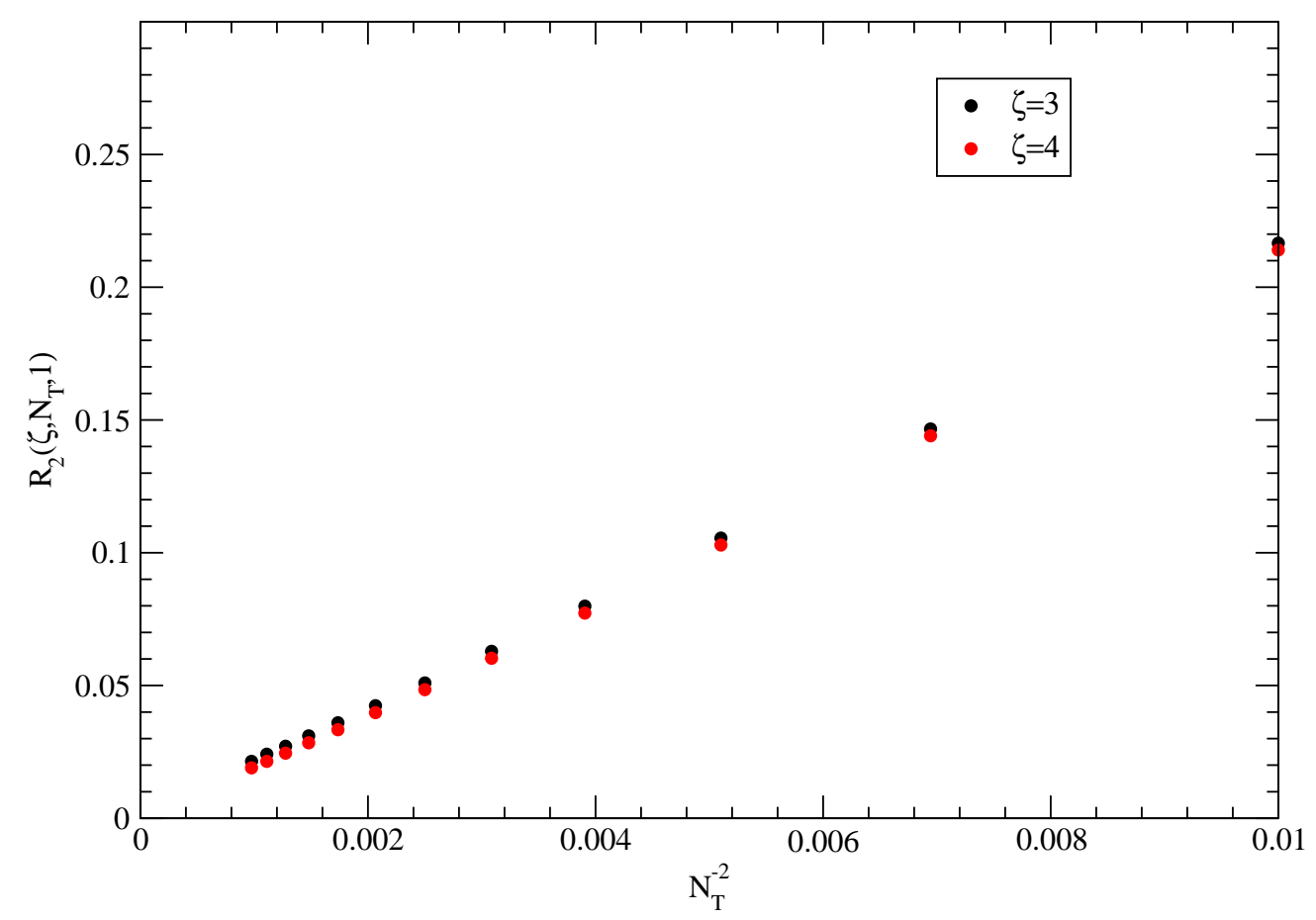

Figure 2: Numerical evidence that $\zeta=4$ is large enough for the computation at hand.

density operator will be

$$
\chi\left(N_{T}, m_{w}\right)=\sum_{j_{1}, j_{2}=1}^{\infty} c_{j_{1}} c_{j_{2}} I_{j_{1}+j_{2}}\left(N_{T}, m_{w}\right) .
$$

As per the conjecture in (5.21), the quark number susceptibility will give the correct $\frac{1}{3 N_{T}^{2}}$ behavior as long as

$$
\sum_{j} c_{j}=1
$$

In order to have no divergence, we need to choose the coefficients, $c_{j}$, such that

$$
\sum_{j_{1}, j_{2}=1}^{\infty} c_{j_{1}} c_{j_{2}} I_{j_{1}+j_{2}}\left(\infty, m_{w}\right)=0
$$

and this is only one condition and we have infinite coefficients. Therefore, we can find a large class of number density operators that have the correct finite behavior in the free fermion limit. Addition of gauge fields cannot give rise to new divergences as long as all couplings and masses are properly renormalized. We could therefore choose 


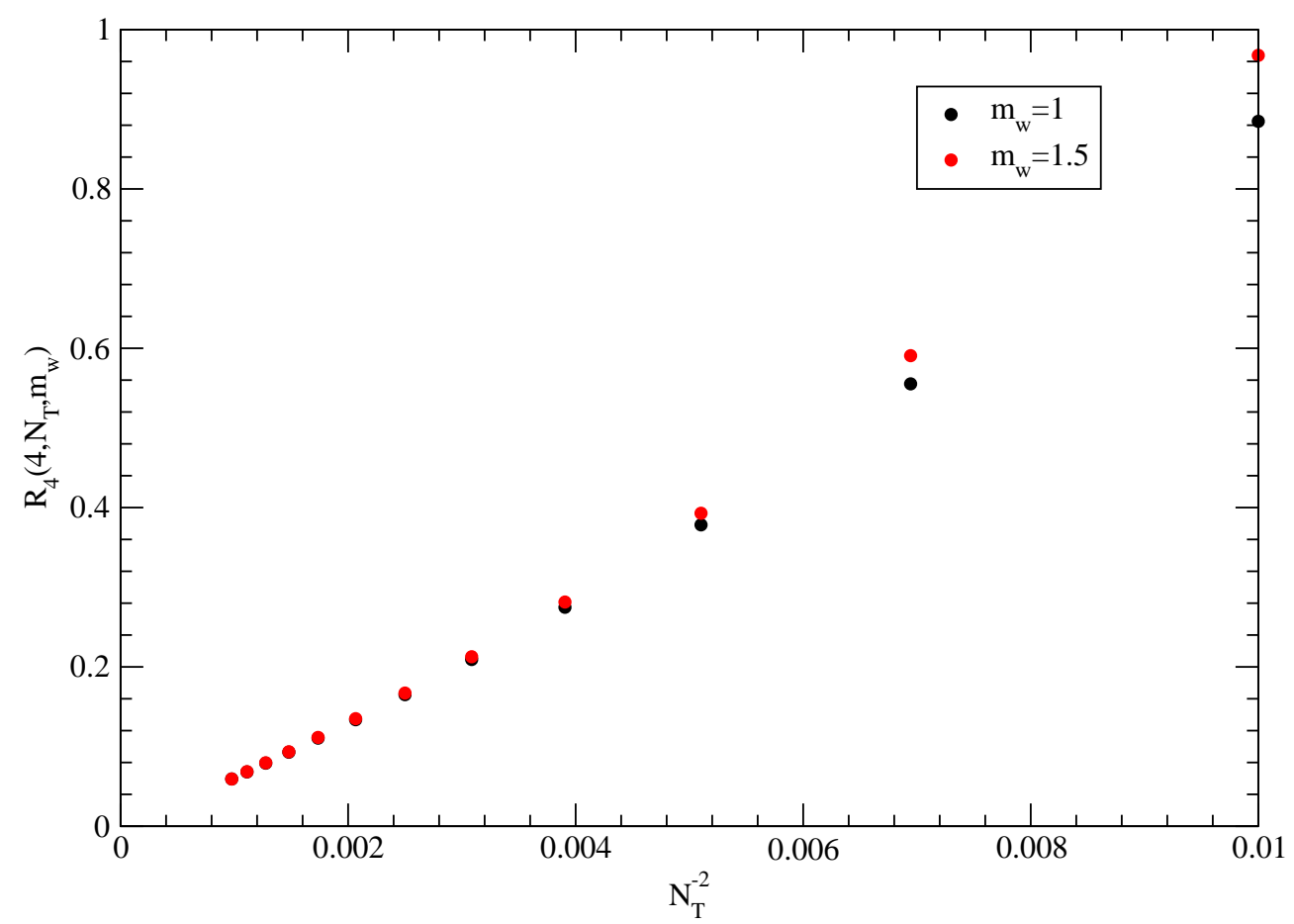

Figure 3: The effect of the Wilson mass parameter on the subleading terms is minimal.

$c_{1}=\alpha$ and $c_{2}=(1-\alpha)$ with $\alpha$ chosen to cancel the free fermion divergence. This will provide one good choice for the number density operator in the full interacting theory. There is clearly one weakness to this approach since one has to tune $\alpha$ to cancel the divergence.

Dynamical simulations of overlap fermions are currently being performed both at zero temperature [21] and finite temperature [22]. It would be interesting to compute the quark number susceptibility in such simulations using the operators presented in this paper and compare them with the complimentary approach of [13]. The two approaches are quite different in their construction. We only couple the chemical potential to the physical fermions. In addition to coupling the chemical potential to the physical fermions, it is also coupled to an infinite number of regulator fields in the Bloch-Wettig scheme. A related issue is the definition of the topological charge. It does not depend on the chemical potential in our scheme contrary to the Bloch-Wettig scheme. The continuum definition of the topological charge based on the counting of the zero modes of the chiral Dirac operator is not affected by the insertion of the chemical potential and our scheme maintains this continuum property on the lattice. Since we expect to work with large values of lattice chemical potential particularly close to the physical transition that separates hadronic 
matter from quark-gluon plasma, the lattice spacing effects arising from the topological charge depending on the chemical potential could be severe. In spite of these differences, we expect lattice simulations to show that the two approaches agree in the continuum limit.

\section{A. Details of the Hermitian Wilson-Dirac operator}

$$
H_{w}=\left(\begin{array}{cc}
B-m_{w} & C \\
C^{\dagger} & -B+m_{w}
\end{array}\right)
$$

be the massive Dirac operator with $0<m_{w}<2$. In the above equation,

$$
\begin{aligned}
B & =\frac{1}{2} \sum_{\mu}\left(2-T_{\mu}-T_{\mu}^{\dagger}\right) \\
C & =\frac{1}{2} \sum_{\mu} \sigma_{\mu}\left(T_{\mu}-T_{\mu}^{\dagger}\right) \\
\left(T_{\mu} \phi\right)(x) & =U_{\mu}(x) \phi(x+\hat{\mu})
\end{aligned}
$$

with

$$
\sigma_{1}=\left(\begin{array}{cc}
0 & 1 \\
1 & 0
\end{array}\right) ; \quad \sigma_{2}=\left(\begin{array}{cc}
0 & -i \\
i & 0
\end{array}\right) ; \sigma_{3}=\left(\begin{array}{cc}
1 & 0 \\
0 & -1
\end{array}\right) ; \quad \sigma_{4}=\left(\begin{array}{cc}
i & 0 \\
0 & i
\end{array}\right)
$$

in $d=4$ and

$$
\sigma_{1}=1 ; \quad \sigma_{2}=-i
$$

in $d=2$. The Dirac matrices in the chiral basis are

$$
\gamma_{\mu}=\left(\begin{array}{cc}
0 & \sigma_{\mu} \\
\sigma_{\mu}^{\dagger} & 0
\end{array}\right) ; \quad \gamma_{5}=\left(\begin{array}{cc}
1 & 0 \\
0 & -1
\end{array}\right)
$$

We will assume that $H_{w}$ is a $2 n \times 2 n$ matrix and we will assume the gauge field is in the zero topological sector. ${ }^{4}$ Let

$$
H_{w} U=U \Lambda
$$

with

$$
U=\left(\begin{array}{cc}
\alpha & \gamma \\
\beta & \delta
\end{array}\right) ; \quad \Lambda=\operatorname{diag}\left(\lambda_{1}^{+}, \cdots, \lambda_{n}^{+},-\lambda_{1}^{-}, \cdots,-\lambda_{n}^{-}\right) .
$$

and $\lambda_{i}^{ \pm}>0$ for all $i$.

\section{B. Details of the derivation of generating functional for massive overlap fermions with a chemical potential}

Like in (3), we can introduce Grassmann variables, $\zeta_{R}, \zeta_{L}, \bar{\zeta}_{R}, \bar{\zeta}_{L}$, that anticommute with all fermionic operators and Grassmann variables to rewrite (4.1) in terms of (3.1) as

$$
Z\left(\bar{\xi}, \xi ; \hat{\mu}_{R}, \hat{\mu}_{L} ; m\right)=\int d \zeta_{L} d \bar{\zeta}_{R} d \bar{\zeta}_{L} d \zeta_{R} e^{\bar{\zeta}_{R} \zeta_{L}-\bar{\zeta}_{L} \zeta_{R}}
$$

\footnotetext{
${ }^{4}$ The overlap formalism is designed to work in all topological sectors and this is one of the important features of the formalism. But, we can restrict ourselves to the zero topological sector to simplify the discussion. All results will trivially extend to all topological sectors.
} 


$$
\begin{aligned}
& {\left[{ }_{R}\langle-| \otimes{ }_{L}\langle+|\right] e^{-\sqrt{m} \bar{\zeta}_{R} d_{R}-\sqrt{m} \zeta_{R} u_{R}^{\dagger}-\sqrt{m} \bar{\zeta}_{L} u_{L}-\sqrt{m} \zeta_{L} d_{L}^{\dagger}}} \\
& e^{\bar{\xi}_{R} d_{R}+\xi_{R} u_{R}^{\dagger}+u_{R}^{\dagger} \hat{\mu}_{R} d_{R}+\xi_{L} d_{L}^{\dagger}+\bar{\xi}_{L} u_{L}-d_{L}^{\dagger} \hat{\mu}_{L} u_{L}}\left[|-\rangle_{L} \otimes|+\rangle_{R}\right] .
\end{aligned}
$$

Using (3.4) the above equation can be written as

$$
\begin{aligned}
Z\left(\bar{\xi}, \xi ; \hat{\mu}_{R}, \hat{\mu}_{L} ; m\right)= & \operatorname{det} \alpha \operatorname{det}\left(1+\hat{\mu}_{R} \beta \alpha^{-1}\right) \operatorname{det} \alpha^{\dagger} \operatorname{det}\left(1-\hat{\mu}_{L}\left(\beta \alpha^{-1}\right)^{\dagger}\right) \\
& \int d \zeta_{L} d \bar{\zeta}_{R} d \bar{\zeta}_{L} d \zeta_{R} \bar{\zeta}_{R} \bar{\zeta}_{L}-\bar{\zeta}_{L} \zeta_{R} \\
& e^{\left(\bar{\xi}_{R}-\sqrt{m} \bar{\zeta}_{R}\right) \frac{1}{\alpha \beta^{-1}+\hat{\mu}_{R}}\left(\xi_{R}-\sqrt{m} \zeta_{R}\right)} \\
& e^{\left(\bar{\xi}_{L}-\sqrt{m} \bar{\zeta}_{L}\right)\left[\frac{1}{\left(\alpha \beta^{-1}\right)^{\dagger}-\hat{\mu}_{L}}\right]\left(\xi_{L}-\sqrt{m} \zeta_{L}\right)} .
\end{aligned}
$$

We can write the exponent in the integrand as

$$
S=\bar{\zeta} F \zeta-\sqrt{m}(\bar{\zeta} G \xi+\bar{\xi} G \zeta)+\bar{\xi} G \xi
$$

where

$$
\bar{\zeta}=\left(\begin{array}{ll}
\bar{\zeta}_{R} & \bar{\zeta}_{L}
\end{array}\right) ; \quad \zeta=\left(\begin{array}{c}
\zeta_{R} \\
\zeta_{L}
\end{array}\right)
$$

and

$$
F=\left(\begin{array}{cc}
m G_{1} & 1 \\
-1 & m G_{2}^{\dagger}
\end{array}\right) ; \quad G=\left(\begin{array}{cc}
G_{1} & 0 \\
0 & G_{2}^{\dagger}
\end{array}\right)
$$

and

$$
G_{1}=\frac{1}{\alpha \beta^{-1}+\hat{\mu}_{R}} ; \quad G_{2}^{\dagger}=\frac{1}{\left(\alpha \beta^{-1}\right)^{\dagger}-\hat{\mu}_{L}} .
$$

Integration of $\bar{\zeta}$ and $\zeta$ yields

$$
\operatorname{det} F e^{\bar{\xi}\left(G-m G F^{-1} G\right) \xi} \text {. }
$$

The second line in the last equality of (4.2) is $\operatorname{det} F$. One can show that

$$
F^{-1}=\left(\begin{array}{cc}
m G_{2}^{\dagger} \frac{1}{1+m^{2} G_{1} G_{2}^{\dagger}} & -\frac{1}{1+m^{2} G_{2}^{\dagger} G_{1}} \\
\frac{1}{1+m^{2} G_{1} G_{2}^{\dagger}} & m G_{1} \frac{1}{1+m^{2} G_{2}^{\dagger} G_{1}}
\end{array}\right),
$$

and it follows that

$$
G-m G F^{-1} G=\left(\begin{array}{cc}
\frac{1}{1+m^{2} G_{1} G_{2}^{\dagger}} G_{1} & G_{1} \frac{m}{1+m^{2} G_{2}^{\dagger} G_{1}} G_{2}^{\dagger} \\
-G_{2}^{\dagger} \frac{m}{1+m^{2} G_{1} G_{2}^{\dagger}} G_{1} & \frac{1}{1+m^{2} G_{2}^{\dagger} G_{1}} G_{2}^{\dagger}
\end{array}\right)
$$

from which the last two lines of (4.2) follows.

\section{Acknowledgments}

R.N. would like to thank the Theory Group at TIFR for two invitations in the past two years that triggered the current collaboration. The authors would like to acknowledge several useful discussions with Rajiv Gavai. R.N. acknowledges partial support by the NSF under grant number PHY-0854744. 


\section{References}

[1] G. Endrodi, Z. Fodor, S. D. Katz, K. K. Szabo, JHEP 1104, 001 (2011). [arXiv:1102.1356 [hep-lat]].

[2] S. Gupta, PoS CPOD2009, 025 (2009). [arXiv:0909.4630 [nucl-ex]].

[3] R. V. Gavai, S. Gupta, Phys. Rev. D78, 114503 (2008). [arXiv:0806.2233 [hep-lat]].

[4] S. Ejiri, F. Karsch, K. Redlich, Phys. Lett. B633, 275-282 (2006). [hep-ph/0509051].

[5] C. R. Allton, S. Ejiri, S. J. Hands, O. Kaczmarek, F. Karsch, E. Laermann, C. Schmidt, L. Scorzato, Phys. Rev. D66, 074507 (2002). [hep-lat/0204010].

[6] R. V. Gavai, S. Gupta, Phys. Rev. D68, 034506 (2003). [hep-lat/0303013].

[7] C. DeTar, L. Levkova, S. Gottlieb, U. M. Heller, J. E. Hetrick, R. Sugar, D. Toussaint, Phys. Rev. D81, 114504 (2010). [arXiv:1003.5682 [hep-lat]].

[8] M. Asakawa, U. W. Heinz, B. Muller, Phys. Rev. Lett. 85, 2072-2075 (2000). [hep-ph/0003169].

[9] R. V. Gavai, S. Gupta, Eur. Phys. J. C43, 31-34 (2005). [hep-ph/0502198].

[10] J. P. Blaizot, E. Iancu, A. Rebhan, Eur. Phys. J. C27, 433-438 (2003). [hep-ph/0206280].

[11] P. Hasenfratz, F. Karsch, Phys. Lett. B125, 308 (1983).

[12] H. Neuberger, Phys. Lett. B 417, 141 (1998) [arXiv:hep-lat/9707022].

[13] J. C. R. Bloch and T. Wettig, Phys. Rev. Lett. 97, 012003 (2006) [arXiv:hep-lat/0604020].

[14] J. C. R. Bloch and T. Wettig, Phys. Rev. D 76, 114511 (2007) [arXiv:0709.4630 [hep-lat]].

[15] H. Neuberger, Phys. Rev. D57, 5417-5433 (1998). [hep-lat/9710089].

[16] R. G. Edwards, U. M. Heller, R. Narayanan, Phys. Rev. D59, 094510 (1999). [hep-lat/9811030].

[17] C. Gattringer, L. Liptak, Phys. Rev. D76, 054502 (2007). [arXiv:0704.0092 [hep-lat]].

[18] D. Banerjee, R. V. Gavai and S. Sharma, Phys. Rev. D 78, 014506 (2008) [arXiv:0803.3925 [hep-lat]].

[19] R. V. Gavai, S. Sharma, Phys. Rev. D81, 034501 (2010). [arXiv:0906.5188 [hep-lat]].

[20] R. Narayanan and H. Neuberger, Nucl. Phys. B 443, 305 (1995) [arXiv:hep-th/9411108].

[21] S. Hashimoto, PoS LATTICE2008, 011 (2008). [arXiv:0811.1257 [hep-lat]].

[22] G. Cossu et al. [ JLQCD Collaboration ], PoS LATTICE2010, 174 (2010). [arXiv:1011.0257 [hep-lat]]. 